\title{
Multi-Area Energy and Reserve Dispatch Under Wind Uncertainty and Equipment Failures
}

Ali Ahmadi-Khatir, Member, IEEE, Antonio J. Conejo, Fellow, IEEE, and Rachid Cherkaoui, Senior Member, IEEE

\begin{abstract}
The cross-border trading among electricity markets in an interconnected multi-area system (e.g., in central Europe) and the integration of renewable resources (e.g., wind energy) have remarkably increased in recent years. To efficiently operate such system, a proper coordination among different areas is required. Within this context, a decentralized algorithm for market clearing is proposed in this paper to dispatch simultaneously energy and reserve under wind generation uncertainty and equipment failures. The proposed technique does not require a central operator but just a moderate interchange of information among neighboring areas. Additionally, the benefit of cross-border trading is studied.
\end{abstract}

Index Terms-Decentralized dispatch, energy and reserve, equipment failure, interconnections, multi-area power system, renewable generation.

\section{NOTATIONS}

The notation is provided below.

\section{1) Indices:}

\section{$A, A A$ \\ $i$ \\ $n, m, r$ \\ $s$}

$$
t
$$

$w$

\section{2) Sets:}

$\Lambda_{A}$

$\Gamma_{A}$
Indices of areas.

Index of generating units.

Indices of area buses.

Index of scenarios $s=(f, \bar{w})$ including failure $(f)$ and wind uncertainty $(\bar{w})$, running from 1 to $S$.

Index of time periods running from 1 to $T$.

Index of wind farms.

Set of internal lines of area A.

Set of tie-lines of area A.
$\Omega_{t s}^{A}$

$\mathcal{G}_{n}^{A}$

$\mathcal{W}_{n}^{A}$

$\mathcal{B}_{n}^{A}$

$\mathcal{N}_{n}^{A}$

3) Parameters:

$C_{i t}^{\mathrm{RU}}$

$C_{i t}^{\mathrm{RD}}$

$D_{n t}^{A}$

$l_{n r}^{A, \max }$

$P_{i}^{\max }$

$P_{w t}^{\mathrm{Offer}}$

$P_{w s t}$

$R_{i}^{\mathrm{U}, \max }$

$R_{i}^{\mathrm{U}, \max }$

$R D_{i}^{\text {down }}$

$R U_{i}^{\mathrm{up}}$

$X_{i s t}^{n}$

$x_{n r}$
$x_{n r s}$
$\pi_{s}$
$\nu_{n t}^{\text {Lost }}$

$x_{n r}$

Manuscript received November 21, 2012; revised March 09, 2013 and Apri 27, 2013; accepted May 27, 2013. This work was supported by Swiss Electric Research for the project of multi-area security assessment. Paper no. TPWRS01293-2012.

A. Ahmadi-Khatir and R. Cherkaoui are with the Power System Research Group, Ecole Polytechnique Federale de Lausanne (EPFL), Lausanne, Switzerland (e-mail: ali.khatir@epfl.ch; rachid.cherkaoui@epfl.ch).

A. J. Conejo is with the Universidad de Castilla-La Mancha, Ciudad Real, Spain (e-mail: antonio.Conejo@uclm.es).

Digital Object Identifier 10.1109/TPWRS.2013.2265854
Set of available generating units in area $\mathrm{A}$ in period $t$ and scenario $s$.

Set of generating units at bus $n$ of area A.

Set of wind farm at bus $n$ of area A.

Set of border buses of other areas connected to bus $n$ in area A.

Set of internal buses connected to bus $n$ in area A.

Price offer of unit $i$ for up reserve in period $t[\$ / \mathrm{MWh}]$.

Price offer of unit $i$ for down reserve in period $t$ [\$/MWh].

Load demand of bus $n$ of area $A$ in period $t$ [MW].

Maximum transfer capability of internal or tie-line $n r$ of area $A$ [MW].

Maximum capacity of unit $i$ [MW].

Wind power offer of farm $w$ in period $t$ [MW].

Realization of wind power production associated with wind farm $w$ in period $t$ and scenario $s$ [MW].

Maximum up reserve that can be provided by unit $i[\mathrm{MW}]$.

Maximum down reserve that can be provided by unit $i$ [MW].

Ramp-down limit of unit $i[\mathrm{MW} / \mathrm{h}]$.

Ramp-up limit of unit $i[\mathrm{MW} / \mathrm{h}]$.

$1 / 0$ binary parameter: 1 if unit $i$ located at bus $n$ in period $t$ and scenario $s$ is out of service and 0 otherwise.

Reactance of line $n r$ [per unit].

Reactance of line $n r$ under scenario $s$ [per unit].

Probability of scenario $s$.

Value of lost load for load $n$ in period $t$ [\$/MWh]. 
4) First-Stage Variables:

$T_{n m, t}^{A} \quad$ Scheduled power flow from $n$ to $m$ through tie-line $n m$ between areas $\mathrm{A}$ and AA in period $t$ [MW].

$P_{i t} \quad$ Scheduled power output of unit $i$ in period $t$ [MW].

$P_{w t} \quad$ Scheduled wind generation of wind farm $w$ in period $t$ [MW].

$R_{i t}^{\mathrm{U}} \quad$ Up reserve scheduled for unit $i$ in period $t$ [MW].

$R_{i t}^{\mathrm{D}} \quad$ Down reserve scheduled for unit $i$ in period $t$ [MW].

$\delta_{n t}^{A} \quad$ Scheduled phase angle of bus $n$ of area A in period $t[\mathrm{rad}]$.

\section{5) Second-Stage Variables:}

$e_{n m, s t}^{A} \quad$ Power from $n$ to $m$ through tie-line $n m$ between areas A and AA in period $t$ and scenario $s[\mathrm{MW}]$.

$L_{n s t}^{\text {shed }} \quad$ Load shedding imposed on load $n$ of area A in period $t$ and scenario $s$ [MW].

$P_{w s t}^{\text {spill }}$

$r_{i s t}^{\mathrm{U}}$

$r_{i s t}^{\mathrm{D}}$

$\theta_{n s t}^{A}$

Wind power spillage of wind farm $w$ in period $t$ and scenario $s[\mathrm{MW}]$.

Up reserve deployed by unit $i$ in period $t$ and scenario $s[\mathrm{MW}]$.

Down reserve deployed by unit $i$ in period $t$ and scenario $s$ [MW].

Phase angle of bus $n$ of area $\mathrm{A}$ in period $t$ and scenario $s$ [rad].

\section{6) Dual Variables:}

$\gamma_{n t}^{A} \quad$ Lagrangian multiplier associated with power balance (1b) pertaining to bus $n$ in period $t$; this corresponds to standard definition of locational marginal price (LMP) at market stage [\$/MW].

$\lambda_{n m, t}^{A}$

Lagrangian multiplier associated with equality constraints (1d) or (2b) pertaining to tie-line $n m$ in period $t$ [\$/MW].

$\mu_{n m, t}^{A} \quad$ Lagrangian multiplier associated with inequality constraints (1e) or (2c) pertaining to tie-line $n m$ in period $t$ [\$/MW].

$\eta_{n m, t}^{A}$ $\gamma_{n s t}^{A}$

$\lambda_{n m, s t}^{A}$

$\mu_{n m, s t}^{A}$

$\eta_{n m, s t}^{A}$
Lagrangian multiplier associated with power balance ( $1 \mathrm{n})$ pertaining to bus $n$ in period $t$ and scenario $s$; this corresponds to definition of LMP at real-time operation [\$/MW].

Lagrangian multiplier associated with equality constraints (1p) or (2e) pertaining to tie-line $n m$ in period $t$ and scenario $s$ [\$/MW].

Lagrangian multiplier associated with inequality constraints (1q) or (2f) pertaining to tie-line $n m$ in period $t$ and scenario $s[\$ / M W]$.

Lagrangian multiplier associated with inequality constraints (1r) or $(2 \mathrm{~g})$ pertaining to tie-line $\mathrm{nm}$ in period $t$ and scenario $s$ [\$/MW].

\section{7) Variables of Neighboring Areas:}

$\widehat{\delta}_{m t}^{A A}$

$\widehat{T}_{m n, t}^{A A}$

$\widehat{\lambda}_{m n, t}^{A A}$

$\widehat{\mu}_{m n, t}^{A A}$

$\widehat{\eta}_{m n, t}^{A A}$

$\widehat{\theta}_{m s t}^{A A}$

$\widehat{e}_{m n, s t}^{A A}$

$\widehat{\lambda}_{m n, s t}^{A A}$

$\widehat{\mu}_{m n, s t}^{A A}$

$\widehat{\eta}_{m n, s t}^{A A} \mathrm{x}$
Phase angle of bus $m$ of neighboring area AA in period $t[\mathrm{rad}]$.

Scheduled power flow from $m$ to $n$ through tie-line $m n$ between areas AA and $\mathrm{A}$ in period $t[\mathrm{MW}]$.

Lagrangian multipliers associated with equality constraints $(2 b)$ pertaining to neighboring area AA $[\$ / M W]$.

Lagrangian multipliers associated with inequality constraints $(2 \mathrm{c})$ pertaining to neighboring area AA [\$/MW].

Lagrangian multipliers associated with inequality constraints (2d) pertaining to neighboring area $\mathrm{AA}[\$ / \mathrm{MW}]$.

Phase angle of bus $m$ of neighboring area AA in period $t$ and scenario $s[\mathrm{rad}]$.

Power from $m$ to $n$ through tie-line $m n$ between areas AA and A in period $t$ and scenario $s$ [MW].

Lagrangian multipliers associated with equality constraints (2e) pertaining to neighboring area $\mathrm{AA}[\$ \mathrm{MW}]$.

Lagrangian multipliers associated with inequality constraints ( $2 \mathrm{f}$ ) pertaining to neighboring area AA $[\$ / M W]$.

Lagrangian multipliers associated with inequality constraints $(2 \mathrm{~g})$ pertaining to neighboring area $\mathrm{AA}[\$ \mathrm{MW}]$.
Note that the above variables pertaining to neighboring areas (denoted by superscript AA) are considered as parameters in (2) once the subproblem associated with area A is solved, and vice versa. 


\section{INTRODUCTION}

\section{A. Motivation and Aim}

$\mathbf{T}$ HE rapid growth of cross-border trading (energy and reserve) among electricity markets in each national (e.g., in central Europe) or regional (e.g., in U.S.) system of an interconnected multi-area system calls for the development of a market-clearing model to ensure a secure and economically efficient operation of each national/regional system and of the interconnected system as a whole.

Ideally, a central operator with access to the data (including technical and market data) of the whole system, could centrally dispatch the system, but for political and technical reasons, this kind of operator is unlikely to be implemented. Hence, a decentralized procedure becomes a necessary option. Such alternative allows the market/system operator (MO/SO) of each interconnected area to clear its market, i.e., to find out the optimal energy and reserve dispatch, independently of the other areas, while interchanging some border information with the neighboring areas. It is relevant to note that the interchange of border information is also used to coordinate the PJM and MISO markets [1].

On the other hand, the integration of wind generation into electric power systems worldwide has been very important. Although wind generation is a relatively cheap form of renewable energy, it is variable and uncertain, which adds to the power system uncertainty and poses significant challenges to the system operation. Thus, in order to represent the system uncertainty, the market-clearing tools need to be revised to provide an efficient and secure framework for electricity trading under and in spite of these uncertainties. The reserve is a commodity traded in the market to counteract unpredictable changes in system conditions. It is worth noting that the cross-border trading in multi-area power system improves the system reliability by sharing the resources (energy and reserve) across areas' boundaries, and it may contribute to the economic efficiency as well.

This paper proposes a decentralized market-clearing model, formulated as a two-stage stochastic programming problem, to account for the system uncertainties, in particular wind production and equipment failures, which affects the power system operation and the cross-border trading. The objective is to maximize the overall social welfare (or to minimize the overall social cost) while preserving dispatch independence of the involved areas. Indeed, the proposed model allows optimally dispatching energy and reserve of each area of a multi-area system in a decentralized manner.

The proposed decentralized algorithm relies on an elaborated instance of the Lagrangian decomposition technique that requires no central coordinator but just interchanges of moderate information among neighboring regions [2]. This decomposition scheme is not oriented to improve the computational efficiency, but rather to preserve the independence of each area in a multi-area electric power system.

\section{B. Literature Review and Contributions}

The literature regarding short-term operations describes three fundamental methodologies to address scheduling and dispatching problems under uncertainty, namely, two-stage stochastic programming [3], chance-constrained optimization [3], and robust optimization [4].

In fact, the technical literature is rich in references using twostage stochastic programming to represent uncertainty in the market-clearing procedure, e.g., [5]-[10]; these studies differ one from another in the way the uncertainty is incorporated in the modeling. For example, [5], [8] and [10] consider only equipment failures (discrete in nature) as scenarios in the model, while references [6], [7] and [9] only consider wind uncertainty (continuous in nature) in their stochastic model.

Chance-constrained optimization is applied to solve the market-clearing problem under uncertainty in [11]-[13]. These studies differ on the way the source of uncertainties is incorporated into the market-clearing model. For instance, [11] deals with demand uncertainty, [12] considers wind power uncertainty, and [13] includes both demand and wind power uncertainties. The chance constraint methodology in [13] is based on the conditional value at risk (CVaR) measure. In robust optimization models, the objective is to clear the market against a plausible worst-case realization of the uncertain parameters [14]-[16].

Although the market scheduling problem in the presence of uncertainty has been analyzed for single-area power systems, it has not been thoroughly investigated in multi-area interconnected systems. Nonetheless, several works considering fully reliable multi-area systems (uncertainty-free) have been reported in the literature using different decomposition algorithms. An application of Lagrangian relaxation to solve a multi-area optimal power flow (OPF) is described in [17], while in [18] and [19] an augmented Lagrangian relaxation procedure is used to solve this problem. In some cases, these decomposition techniques may present drawbacks, such as convergence rates that depend on the correct choice of the values for several parameters, or requiring the intervention of a central agent to update information. In [20] the optimality condition decomposition algorithm is used to solve the multi-area OPF problem. This algorithm is simple and efficient, and avoid the aforementioned drawbacks.

Note that problems in which Lagrangian relaxation is useful include unit commitment and economic dispatch [21]-[23].

This paper relies on the decomposition technique reported in [20] for fully exploiting the structure of a multi-area problem to achieve single-area problems that can be solved independently.

The contribution of this paper is threefold:

1) To provide a two-stage stochastic market-clearing model for a multi-area power market able to optimally dispatch energy and reserve, while facilitating the cross-border trading among areas.

2) To determine the optimal mix of up/down reserves as preventive actions, and wind spillage and load shedding as corrective actions in a multi-area interconnected system operating under uncertainty, particularly wind production and equipment (generation units and transmission lines) failures.

3) To develop a simple and efficient decomposition algorithm to solve the proposed stochastic model in a decentralized manner, without the need of a central coordinator and with 
minimal border information interchanged among neighboring areas.

\section{Paper Organization}

The rest of the paper is organized as follows. Section II is the core of this paper providing the mathematical formulation of the market-clearing model in both centralized (Section II-A) and decentralized forms (Section II-B). Section II-C discusses the decomposition algorithm convergence properties, the information required to be interchanged among areas and how this algorithm can be implemented in practice. Section III demonstrates and discusses the results obtained from a two-area three-bus test system and the three-area IEEE-RTS. The conclusions drawn from this study are provided in Section IV.

\section{MARKet-Clearing Model}

The market-clearing model formulated below is intended for simultaneously dispatching energy and reserve in a multi-area interconnected system with cross-border trading under wind production uncertainty and equipment failures. The model corresponds to an auction inspired by those used in most European electricity markets, which leave the on/off commitment decisions to the producers that own the production units [24]-[26]. This multi-area market-clearing auction is cast as a two-stage stochastic programming problem, where the first stage represents the dispatch in the electricity market (e.g., day-ahead schedule), and the second stage represents the actual operation under different scenarios.

Note that the key point of the proposed market-clearing model is to incorporate as a prognosis information on "all possible" real-time outcomes (i.e., plausible realizations of uncertain wind generation and equipment failures) via different scenarios before the uncertain events materialize. This way, we compute the optimal pre-positioning of the slow and inflexible conventional units in the day-ahead market, so that the overall expected social cost is minimized and feasibility is guaranteed for all possible real-time outcomes.

The data required for market clearing are the data that the producers and consumers submit to the corresponding operators, i.e., their offer and bid curves for energy and reserve. In turn, the operators clear the market and set prices and schedules. For clarity, the main assumptions of the proposed market model are summarized below.

1) Loads are assumed to be inelastic and they do not participate in the reserve market.

2) The minimum power output of each generating unit is assumed to be zero; this way, non-convexity issues are sidestepped. Note that this assumption is predominant in European electricity markets.

3) The network is explicitly modeled through a linear dc approximation; network losses are neglected. Note that using the piecewise linear model in [27], losses can be incorporated in the proposed model.

4) Wind power producers offer their forecast production at zero price.

5) The offer curves of generating units are considered quadratic functions, i.e., $C_{i}\left(P_{i t}\right)=c_{i} P_{i t}^{2}+b_{i} P_{i t}+a_{i}$.

6) The time horizon is one day divided in 24 hours.
7) Uncertainties include wind production and equipment failure.

8) Equipment failure occurs during the first period (i.e., $t=$ 1 ), and this equipment will be unavailable for the rest of the scheduling horizon. This way, the time interval by which the contingency scenarios are characterized and thus the non-anticipativity constraints are avoided.

9) The operators of the interconnected areas need to agree on the scenarios (involving wind production and equipment failures) to be considered to clear the market as they need to interchange information per scenario.

Note that most of the above assumptions can easily be relaxed.

The market-clearing model that results from these assumptions translates into a quadratic programming problem. In what follows both the centralized and decentralized formulations are described in detail.

\section{A. Centralized Formulation}

The proposed centralized model is formulated as

$$
\begin{aligned}
& \underset{\Xi}{\operatorname{Minimize}} \\
& \sum_{t} \sum_{t}\left\{\sum_{i \in A}\left[C_{i}\left(P_{i t}\right)+C_{i t}^{\mathrm{RU}} R_{i t}^{\mathrm{U}}+C_{i t}^{\mathrm{RD}} R_{i t}^{\mathrm{D}}\right]+\sum_{s} \pi_{s}\right. \\
& \quad \times \sum_{i \in A}\left[C_{i}\left(P_{i t}+r_{i s t}^{\mathrm{U}}-r_{i s t}^{\mathrm{D}}\right)-C_{i}\left(P_{i t}\right)\right] \\
& \left.\quad+\sum_{s} \pi_{s} \sum_{n \in A}\left[\nu_{n t}^{\text {Lost }} L_{n s t}^{\text {shed }}\right]\right\}
\end{aligned}
$$

subject to :

$$
\begin{aligned}
& \left\{\sum_{i \in \mathcal{G}_{n}^{A}} P_{i t}+\sum_{w \in \mathcal{W}_{n}^{A}} P_{w t}-D_{n t}^{A}-\sum_{r \in \mathcal{N}_{n}^{A}} \frac{1}{x_{n r}}\left(\delta_{n t}^{A}-\delta_{r t}^{A}\right)\right. \\
& -\sum_{m \in \mathcal{B}_{n}^{A}} T_{n m, t}^{A}=0: \gamma_{n t}^{A}, \quad \forall n \in A \\
& \left|\frac{1}{x_{n r}}\left(\delta_{n t}^{A}-\delta_{r t}^{A}\right)\right| \leq l_{n r}^{A, \max }, \quad \forall(n, r) \in \Lambda^{A} \\
& \frac{1}{x_{n m}}\left(\delta_{n t}^{A}-\delta_{m t}^{A A}\right)-T_{n m, t}^{A}=0: \lambda_{n m, t}^{A}, \\
& \forall(n, m) \in \Gamma^{A} \\
& \frac{-1}{x_{n m}}\left(\delta_{n t}^{A}-\delta_{m t}^{A A}\right) \geq-l_{n m}^{A, \max }: \mu_{n m, t}^{A}, \\
& \forall(n, m) \in \Gamma^{A} \\
& \frac{1}{x_{n m}}\left(\delta_{n t}^{A}-\delta_{m t}^{A A}\right) \geq-l_{n m}^{A, \max }: \eta_{n m, t}^{A}, \\
& \forall(n, m) \in \Gamma^{A} \\
& P_{i t}-R_{i t}^{\mathrm{D}} \geq 0, \quad \forall i \in A \\
& P_{i t}+R_{i t}^{\mathrm{U}} \leq P_{i}^{\max }, \quad \forall i \in A \\
& P_{w t} \leq P_{w t}^{\text {Offer }}, \quad \forall w \in A \\
& P_{i t}-P_{i(t-1)} \leq R U_{i}^{\text {up }}, \quad \forall i \in A \\
& P_{i(t-1)}-P_{i t} \leq R D_{i}^{\text {down }}, \quad \forall i \in A \\
& R_{i t}^{\mathrm{U}} \leq R_{i}^{\mathrm{U}, \max }, \quad \forall i \in A \\
& R_{i t}^{\mathrm{D}} \leq R_{i}^{\mathrm{D}, \max }, \quad \forall i \in A
\end{aligned}
$$




$$
\begin{aligned}
& \sum_{i \in \mathcal{G}_{n}^{A}}\left(r_{i s t}^{\mathrm{U}}-r_{i s t}^{\mathrm{D}}\right)+\sum_{w \in \mathcal{W}_{n}^{A}}\left(P_{w s t}-P_{w s t}^{\mathrm{spill}}-P_{w t}\right) \\
& +L_{n s t}^{\mathrm{shed}}-\sum_{r \in \mathcal{N}_{n}^{A}} \frac{1}{x_{n r s}}\left(\theta_{n s t}^{A}-\theta_{r s t}^{A}\right)-\sum_{m \in \mathcal{B}_{n}^{A}} e_{n m, s t}^{A} \\
& =\left(-\sum_{m \in \mathcal{B}_{n}^{A}} T_{n m, t}^{A}-\sum_{r \in \mathcal{N}_{n}^{A}} \frac{1}{x_{n r}}\left(\delta_{n t}^{A}-\delta_{r t}^{A}\right)\right) \\
& \times\left(1-\sum_{i \in \mathcal{G}_{n}^{A}} X_{i s t}^{n}\right) \\
& -\left(\sum_{\substack{j \in \mathcal{G}_{n}^{A} \\
j \neq i}} P_{j s t}+\sum_{w \in \mathcal{W}_{n}^{A}} P_{w t}-D_{n t}^{A}\right) \\
& \times \sum_{i \in \mathcal{G}_{n}^{A}} X_{i s t}^{n}: \gamma_{n s t}^{A}, \quad \forall n \in A, \forall s \\
& \left|\frac{1}{x_{n r s}}\left(\theta_{n s t}^{A}-\theta_{r s t}^{A}\right)\right| \leq l_{n r}^{A, \max }, \\
& \forall(n, r) \in \Lambda^{A}, \forall s \\
& \frac{1}{x_{n m s}}\left(\theta_{n s t}^{A}-\theta_{m s t}^{A A}\right)-e_{n m, s t}^{A}=0: \lambda_{n m, s t}^{A} \\
& \forall(n, m) \in \Gamma^{A}, \quad \forall s \\
& \frac{-1}{x_{n m s}}\left(\theta_{n s t}^{A}-\theta_{m s t}^{A A}\right) \geq-l_{n m}^{A, \max }: \mu_{n m, s t}^{A} \text {. } \\
& \forall(n, m) \in \Gamma^{A}, \forall s \\
& \frac{1}{x_{n m s}}\left(\theta_{n s t}^{A}-\theta_{m s t}^{A A}\right) \geq-l_{n m}^{A, \max }: \eta_{n m, s t}^{A} \text {, } \\
& \forall(n, m) \in \Gamma^{A}, \forall s \\
& P_{w s t}^{\text {spill }} \leq P_{w s t}, \quad \forall s, \forall w \in A \\
& L_{n s t}^{\text {shed }} \leq D_{n t}^{A}, \quad \forall s, \forall n \in A \\
& r_{i s t}^{\mathrm{U}} \leq R_{i t}^{\mathrm{U}}, \quad \forall s, \forall i \in \Omega_{t s}^{A} \\
& r_{i s t}^{\mathrm{D}} \leq R_{i t}^{\mathrm{D}}, \quad \forall s, \forall i \in \Omega_{t s}^{A} \\
& r_{i s t}^{\mathrm{U}} \leq 0, r_{i s t}^{\mathrm{D}} \leq 0, \quad \forall s, \forall i \notin \Omega_{t s}^{A} \\
& P_{i t}, R_{i t}^{\mathrm{U}}, R_{i t}^{\mathrm{D}} \geq 0, \quad \forall i \in A ; P_{w t} \geq 0, \quad \forall w \in A ; \\
& r_{i s t}^{\mathrm{U}}, r_{i s t}^{\mathrm{D}} \geq 0, \quad \forall s, \quad \forall i \in A ; P_{w s t}^{\mathrm{spill}} \geq 0, \\
& \forall s, \forall w \in A ; \\
& L_{n s t}^{\text {shed }} \geq 0, \quad \forall s, \quad \forall n \in A \\
& \delta_{n t}^{A}=0,(n=1) ;\left|\delta_{n t}^{A}\right| \leq \pi, \quad \forall n \in A ; \\
& \theta_{n s t}^{A}=0,(n=1), \quad \forall s ;\left|\theta_{n s t}^{A}\right| \leq \pi, \\
& \forall s, \quad \forall n \in A\} \forall t, \forall A \text {. }
\end{aligned}
$$

The optimization variables of problem $\Xi=$ $\left\{P_{i t}, R_{i t}^{\mathrm{D}}, R_{i t}^{\mathrm{U}}, r_{i s t}^{\mathrm{U}}, r_{i s t}^{\mathrm{D}}, \forall i, \forall s ; P_{w t}, P_{w s t}^{\mathrm{spill}}, \forall w, \forall s ;\right.$ $\left.\delta_{n t}^{A}, \theta_{n s t}^{A}, L_{n s t}^{\text {shed }}, \forall n, \forall s ; T_{n m, t}^{A}, e_{n m, s t}^{A}, \forall(n, m) \in \Gamma^{A}, \forall s\right\}$ $\forall t, \forall A$. This variable set encompasses both first-stage variables, i.e., those variables that do not depend on any particular scenario realization and that represent hereand-now decisions; and second-stage variables, i.e., those variables pertaining to each particular scenario that that represent wait-and-see decisions.
Observe that the proposed model is a single-shot marketclearing model that incorporates a prognosis of real-time outcomes and that it is solved in a single point in time. Needless to say, such market clearing needs the follow up of a real-time market.

The objective function (1a) is the expected cost of reserve and energy supply, which includes the costs pertaining to the market stage (simultaneous dispatch of energy and reserve) and the costs incurred in the real-time operation (reserve deployment and load shedding actions).

Three sets of constraints are included in the formulation: the first-stage constraints modeling the functioning of the market (1b)-(1m), the second-stage constraints modeling the actual operation of the system once a particular scenario $s$ is realized (1n)-(1t), and finally, the linking constraints (1u)-(1w), which bind the market decisions to the actual operation. Notice that, from a stochastic programming perspective, the linking constraints are needed to relate first-and second-stage variables.

Constraints (1b) are market balance equations. Constraints (1c) ensure the transmission capacity limits of the internal lines of each area. Constraints (1d) represent the tie-line power flow in market scheduling. The set of constraints (1e) and (1f) enforces the capacity limit of tie-lines of each area. The set of constraints (1g) and (1h) implies that the power plus the reserves scheduled in the market must be between the minimum power output and the capacity of the unit. Constraints (1i) limit the market schedule of wind generation to its production offer. Inter-temporal constraints (ramping up and ramping down limits) are enforced by (1j) and (1k). Constraints (11) and (1m) guarantee that the up/down scheduled reserves at the market stage for each generating unit do not exceed its reserve capacity offers (i.e., $R_{i}^{\mathrm{U}, \max }$ and $R_{i}^{\mathrm{D}, \max }$ ). In this sense, the reserve capacity offers are consistent with the physical limits of the generating unit. Constraints (1n) are power balance equations for border and interior buses in scenario states. These constraints guarantee that any scenario realization is made feasible by deploying reserve, wind spillage, load shedding and/or the assistance coming from neighboring areas. Please note that the binary parameter $X_{i s t}^{n}$ is used to describe single unit outages, whereas a line outage is modeled by setting the parameter $x_{n m s}$ to infinity. Constraints (10) ensure that flows in all available interior lines under scenario $s$ are below their capacity limits. Constraints (1p) represent the tie-line power flow during real time operation. The set of constraints (1q)-(1r) enforces capacity limits for all available tie-lines and all scenarios. It is emphasized that the parameter $x_{n m s}$ is equal to $\infty$ for any scenario $s$ in which line $n m$ is out of service. Constraints (1s) and (1t) set bound on the amount of wind power that is spilled and on the amount of load that is involuntarily shed, respectively, under each scenario $s$. Constraints (1u)-(1v) state that the amount of up/down reserve that the available generating units can deploy is limited to the quantity established in the market. Constraints (1w) imply that unit $i$ cannot deploy up/down reserves if it is not available, i.e., if $i \notin \Omega_{t s}^{A}$ in period $t$ and scenario $s$. Constraints $(1 \mathrm{x})$ declare the nonnegative nature of certain variables. The series of constraints (1y) constitutes the variables declarations for phase angles both at the market stage and in real-time operation. 


\section{B. Decentralized Formulation}

In the decentralized formulation, particular attention should be paid to constrains (1d), (1e), (1f), (1p), (1q) and (1r). They are commonly known as complicating constraints, since these equations contain variables from more than one area (identified through superscripts A and AA) and prevent each area from operating independently from the others.

The algorithm principle presented in [20] is employed in this paper to decompose the problem per area by relaxing all the complicating constraints pertaining to areas different than A (i.e. dualize them to the objective function of problem (1)), but maintaining the own complicating constraints. It is formulated as

$$
\begin{aligned}
& \operatorname{Minimize}_{\Xi_{A}} \\
& \sum_{t}\left\{\sum_{i \in A}\left[C_{i}\left(P_{i t}\right)+C_{i t}^{\mathrm{RU}} R_{i t}^{\mathrm{U}}+C_{i t}^{\mathrm{RD}} R_{i t}^{\mathrm{D}}\right]\right. \\
& +\sum_{(n, m) \in \Gamma^{A}} \widehat{\lambda}_{m n, t}^{A A}\left[\frac{1}{x_{m n}}\left(\widehat{\delta}_{m t}^{A A}-\delta_{n t}^{A}\right)-\widehat{T}_{m n, t}^{A A}\right] \\
& +\sum_{(n, m) \in \Gamma^{A}} \widehat{\mu}_{m n, t}^{A A}\left[\frac{-1}{x_{m n}}\left(\widehat{\delta}_{m t}^{A A}-\delta_{n t}^{A}\right)+l_{m n}^{A A, \max }\right] \\
& +\sum_{(n, m) \in \Gamma^{A}} \widehat{\eta}_{m n, t}^{A A}\left[\frac{1}{x_{m n}}\left(\widehat{\delta}_{m t}^{A A}-\delta_{n t}^{A}\right)+l_{m n}^{A A, \max }\right] \\
& +\sum_{s} \pi_{s} \sum_{i \in A}\left[C_{i}\left(P_{i t}+r_{i s t}^{\mathrm{U}}-r_{i s t}^{\mathrm{D}}\right)-C_{i}\left(P_{i t}\right)\right] \\
& +\sum_{s} \pi_{s} \sum_{n \in A}\left[\nu_{n}^{\text {Lost }} L_{n s t}^{\text {shed }}\right] \\
& +\sum_{s} \sum_{(n, m) \in \Gamma^{A}} \widehat{\lambda}_{m n, s t}^{A A} \\
& \times\left[\frac{1}{x_{m n s}}\left(\widehat{\theta}_{m s t}^{A A}-\theta_{n s t}^{A}\right)-\widehat{e}_{m n, s t}^{A A}\right] \\
& +\sum_{s} \sum_{(n, m) \in \Gamma^{A}} \widehat{\mu}_{m n, s t}^{A A} \\
& \times\left[\frac{-1}{x_{m n s}}\left(\widehat{\theta}_{m s t}^{A A}-\theta_{n s t}^{A}\right)+l_{m n}^{A A, \max }\right] \\
& +\sum_{s} \sum_{(n, m) \in \Gamma^{A}} \widehat{\eta}_{m n, s t}^{A A} \\
& \left.\times\left[\frac{1}{x_{m n s}}\left(\widehat{\theta}_{m s t}^{A A}-\theta_{n s t}^{A}\right)+l_{m n}^{A A, \max }\right]\right\}
\end{aligned}
$$

subject to :

$$
\begin{aligned}
& \frac{1}{x_{n m}}\left(\delta_{n t}^{A}-\widehat{\delta}_{m t}^{A A}\right)-T_{n m, t}^{A}=0: \lambda_{n m, t}^{A}, \\
& \forall(n, m) \in \Gamma^{A}, \quad \forall t \\
& \frac{-1}{x_{n m}}\left(\delta_{n t}^{A}-\widehat{\delta}_{m t}^{A A}\right) \geq-l_{n m}^{A, \max }: \mu_{n m, t}^{A} \\
& \forall(n, m) \in \Gamma^{A}, \quad \forall t \\
& \frac{1}{x_{n m}}\left(\delta_{n t}^{A}-\widehat{\delta}_{m t}^{A A}\right) \geq-l_{n m}^{A, \max }: \eta_{n m, t}^{A} \\
& \forall(n, m) \in \Gamma^{A}, \quad \forall t \\
& \frac{1}{x_{n m s}}\left(\theta_{n s t}^{A}-\widehat{\theta}_{m s t}^{A A}\right)-e_{n m, s t}^{A}=0: \lambda_{n m, s t}^{A} \\
& \forall(n, m) \in \Gamma^{A}, \quad \forall s, \quad \forall t
\end{aligned}
$$

$$
\begin{aligned}
& \frac{-1}{x_{n m s}}\left(\theta_{n s t}^{A}-\widehat{\theta}_{m s t}^{A A}\right) \geq-l_{n m}^{A, \max }: \mu_{n m, s t}^{A} \\
& \forall(n, m) \in \Gamma^{A}, \quad \forall s, \quad \forall t \\
& \frac{1}{x_{n m s}}\left(\theta_{n s t}^{A}-\widehat{\theta}_{m s t}^{A A}\right) \geq-l_{n m}^{A, \max }: \eta_{n m, s t}^{A} \\
& \forall(n, m) \in \Gamma^{A}, \quad \forall s, \quad \forall t \\
& (1 \mathrm{~b})-(1 \mathrm{c}),(1 \mathrm{~g})-(1 \mathrm{o}),(1 \mathrm{~s})-(1 \mathrm{y}) .
\end{aligned}
$$

The optimization variables of problem (2) are those in set $\Xi_{A}=$ $\left\{P_{i t}, R_{i t}^{\mathrm{D}}, R_{i t}^{\mathrm{U}}, r_{i s t}^{\mathrm{U}}, r_{i s t}^{\mathrm{D}}, \forall i, \forall s ; P_{w t}, P_{w s t}^{\mathrm{spill}}, \forall w, \forall s ; \delta_{n t}^{A}, \theta_{n s t}^{A}\right.$, $\left.L_{n s t}^{\mathrm{shed}}, \forall n, \forall s ; T_{n m, t}^{A}, e_{n m, s t}^{A}, \forall(n, m) \in \Gamma^{A}, \forall s\right\} \forall t$.

Note that $\lambda_{n m t}^{A}, \mu_{n m, t}^{A}$ and $\eta_{n m, t}^{A}$ are dual variables related to the equality and inequality constraints pertaining to border lines (complicating constraints) at market level. Similarly, $\lambda_{n m, s t}^{A}$, $\mu_{n m, s t}^{A}$ and $\eta_{n m, s t}^{A}$ are dual variables related to the equality and inequality constraints pertaining to border lines (complicating constraints) at the actual operation. Variables with "hat" identify other areas' variables that are treated as constants with the values they were assigned in the previous iteration of the proposed algorithm.

\section{On Convergence, Information Interchange, and Implementation}

The proposed decentralized algorithm relies on the decoupling of the first-order Karush-Kuhn-Tucker (KKT) optimality conditions of the original problem in such a way that the combination of the KKT conditions of all area sub-problems are identical to the KKT conditions of the original problem. Based on this observation, the decoupled solution of the centralized problem (1) can be achieved using an iterative algorithm. Note that the decomposed nature of the proposed algorithm makes it suitable for large-scale applications. Moreover, under mild convexity assumption, the algorithm is guaranteed to converge. More details on convergence conditions can be found in [28].

At the end of each iteration $\nu$, each area A interchanges with neighboring areas two types of data: 1) border-bus state variables, i.e., voltage angles of border buses under market and real-time operation, $\left(\widehat{\delta}_{n t}^{A}\right)^{\nu+1}=$ $\left(\delta_{n t}^{A}\right)^{\nu}$ and $\left(\widehat{\theta}_{n s t}^{A}\right)^{\nu+1}=\left(\theta_{n s t}^{A}\right)^{\nu}$, and 2) dual data related to border constraints in both market and real-time operation, $\left\{\left(\widehat{\lambda}_{n m, t}^{A}\right)^{\nu+1}=\left(\lambda_{n m, t}^{A}\right)^{\nu},\left(\widehat{\mu}_{n m t}^{A},\right)^{\nu+1}=\right.$ $\left.\left(\mu_{n m, t}^{A}\right)^{\nu},\left(\widehat{\eta}_{n m, t}^{A}\right)^{\nu+1}=\left(\eta_{n m, t}^{A}\right)^{\nu}\right\}$ and $\left\{\left(\widehat{\lambda}_{n m, s t}^{A}\right)^{\nu+1}=\right.$ $\left(\lambda_{n m, s t}^{A}\right)^{\nu},\left(\widehat{\mu}_{n m, s t}^{A}\right)^{\nu+1}=\left(\mu_{n m, s t}^{A}\right)^{\nu},\left(\widehat{\eta}_{n m, s t}^{A}\right)^{\nu+1}=$ $\left.\left(\eta_{n m, s t}^{A}\right)^{\nu}\right\}$. Observe that the interchanged information is moderate and no central coordinator is required.

In terms of interchanged information at each iteration, the decentralized problem (2) can be implemented either in a parallel fashion, i.e., areas solve their respective problems and then interchange border information, or sequentially, i.e., areas are considered sequentially, and as the border information is updated, it becomes available to other areas. We consider below the sequential algorithm whose scheme is presented in Fig. 1.

It is important to note that the convergence behavior of the algorithm is directly influenced by the way in which the border bus states are initialized. Thus, it is advantageous to relax the tolerances at the first iteration and to modify it by considering neighboring data as variables instead of fixed parameters [29]. 
1) Consider $\left\{A_{1}, \cdots, A_{N}\right\}$ as the set of areas. Choose initial values for border-bus states and dual data related to the border constraints. Set counter $v=0$.

2) Set counter $v \leftarrow v+1$ and $j=1$.

3) Solve sub-problem (2) for each area $A_{j}$. Interchange border information (state and dual data) with adjacent areas.

4) If $A_{j} \neq A_{N}$, set $j \leftarrow j+1$ and go to step 3). Otherwise, continue.

5) If $\left.\sum_{(A, n, t)}\left\{\|\left(\lambda_{n m, t}^{A}\right)^{v-(} \lambda_{n m, t}^{A}\right)^{v-1}\|+\|\left(\mu_{n m, t}^{A}\right)^{v-(} \mu_{n m, t}^{A}\right)^{v-1} \|+$

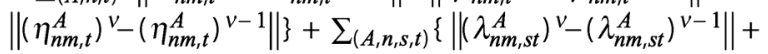

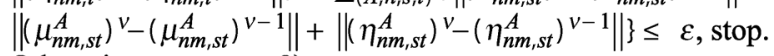
Otherwise, go to step 2).

Fig. 1. Decentralized market-clearing algorithm (sequential form).

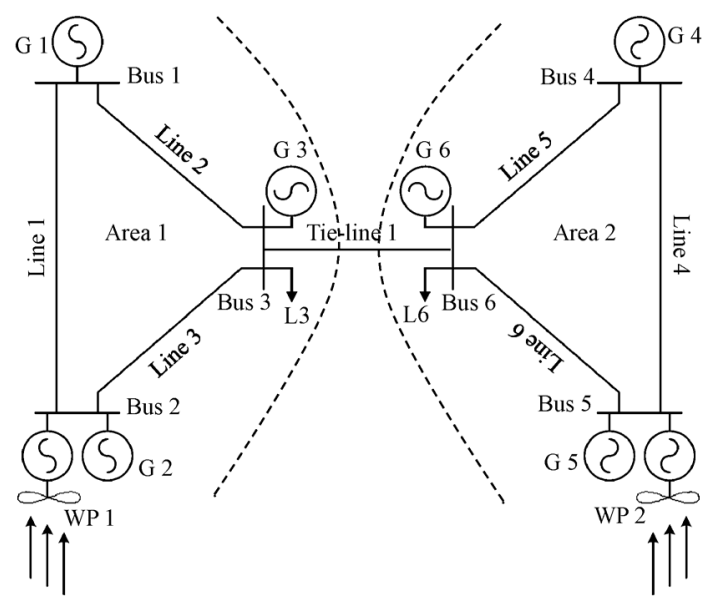

Fig. 2. Six-bus interconnected test system.

In particular, the variable set of optimization problem (2) is extended from $\left\{\Xi_{A}\right\}$ to $\left\{\Xi_{A}, \delta_{m t}^{A A}, \theta_{m s t}^{A A}\right\}$ just for the first iteration and the first area in the sequential algorithm. Note that only border-bus state information is interchanged in the first iteration. For the rest of iterations, the algorithm is run as indicated in Fig. 1.

\section{Illustrative EXAMPLE AND CASE StUdy}

To illustrate the effectiveness of the proposed multi-area market-clearing model, a six-bus interconnected test system and the three-area IEEE-RTS are considered. The simulations presented have been carried out using CPLEX 12.1.0 under GAMS [30] on a Sun Fire X4600M2 with 8 Quad-Core processors running at $2.9 \mathrm{GHz}$ and $256 \mathrm{~GB}$ of RAM.

\section{A. Six-Bus Interconnected Test System}

The considered test system is depicted in Fig. 2. There are two similar areas interconnected by one tie-line. Reactances of the internal lines and the tie-line are all 0.13 p.u. on a base of 100 MVA. The capacities of all internal lines are equal to $100 \mathrm{MW}$, and the capacity of the tie-line is set to $50 \mathrm{MW}$.

Data for the conventional generating units of area 1 are given in Table I. To impose power imports on area 2, the cost functions of generators of area 2 for energy and reserve are doubled, but
TABLE I

Conventional Generating Units Data-AREa 1

\begin{tabular}{c|ccc}
\hline Generator $i$ & 1 & 2 & 3 \\
\hline$P_{i}^{\max }(\mathrm{MW})$ & 100 & 100 & 100 \\
\hline$a_{i}(\$ / \mathrm{h})$ & 100 & 100 & 100 \\
\hline$b_{i}(\$ / \mathrm{MWh})$ & 30 & 40 & 20 \\
\hline$c_{i}\left(\$ / \mathrm{MW}^{2} \mathrm{~h}\right)$ & 0.3 & 0.8 & 0.2 \\
\hline$C_{i t}^{\mathrm{RU}}(\$ / \mathrm{MW} \& \mathrm{~h})$ & 3 & 4 & 2 \\
\hline$C_{i t}^{\mathrm{RD}}(\$ / \mathrm{MW} \& \mathrm{~h})$ & 3 & 4 & 2 \\
\hline
\end{tabular}

TABLE II

HOURLY LOAD DATA

\begin{tabular}{ccccc}
\hline Period t & 1 & 2 & 3 & 4 \\
\hline L3 $(\mathrm{MW})$ & 90 & 110 & 140 & 100 \\
\hline L6 $(\mathrm{MW})$ & 90 & 110 & 140 & 100 \\
\hline
\end{tabular}

TABLE III

WIND POWER SCENARIOS (IN MW)

\begin{tabular}{c|cccc}
\hline \multirow{2}{*}{ Period t } & \multicolumn{4}{|c}{ Wind Scenarios } \\
\cline { 2 - 5 } & $(\mathrm{H}, \mathrm{H})$ & $(\mathrm{M}, \mathrm{M})$ & $(\mathrm{L}, \mathrm{L})$ & $(\mathrm{H}, \mathrm{L})$ \\
\hline 1 & $(10,10)$ & $(5,5)$ & $(0,0)$ & $(10,0)$ \\
\hline 2 & $(30,30)$ & $(20,20)$ & $(10,10)$ & $(30,10)$ \\
\hline 3 & $(40,40)$ & $(25,25)$ & $(15,15)$ & $(40,15)$ \\
\hline 4 & $(20,20)$ & $(10,10)$ & $(5,5)$ & $(20,5)$ \\
\hline
\end{tabular}

the technical data of this area are the same as in area 1. For the sake of simplicity, it is also assumed that the energy and reserve offers of the units in both areas remain unchanged throughout the scheduling horizon. Finally, the units' ramping capabilities and the bounds on the amounts of reserve services offered are set to be the largest possible. Two wind power plants (WP) are located at bus 2 and bus 5 as indicated in Fig. 2. The forecast production of wind generators for market scheduling is $30 \mathrm{MW}$.

The system is tested for a four-hour dispatching horizon where the forecast demands of loads, which are located at buses 3 and 6 , vary hour by hour according to the pattern detailed in Table II. The value of lost load associated with these load demands is assumed to be $1000 \$ / \mathrm{MWh}$.

The data provided so far for this small-scale six-bus test system defines the base case.

We examine below the combined impact of equipment failures and wind production uncertainty on the energy and reserve dispatch of this base case. To this end, we consider the four wind production scenarios listed in Table III, identified as "both areas high $(\mathrm{H}, \mathrm{H})$ ", "both areas medium $(\mathrm{M}, \mathrm{M})$ ", "both areas low $(\mathrm{L}, \mathrm{L})$ " and "area 1 high and area 2 low $(\mathrm{H}, \mathrm{L})$ ", with the same probability of occurrence, 0.25 . In addition, three scenarios including no-contingency scenario, failure of unit G6 and the outage of line 5 in area 2 are taken into consideration. For the sake of simplicity, other equipments in this system are assumed fully reliable, i.e., they never fail. The time between two consecutive failures of these two elements follows an exponential distribution characterized by a MTTF equal to $200 \mathrm{~h}$. The probabilities of occurrence associated with these contingency scenarios are $0.96,0.02$ and 0.02 , in that order. The detail computation of these values can be found in [31]. Thus, each scenario $s$ in the market-clearing formulation is indeed constituted by a pair of a failure event $f$ and a wind production realization $\bar{w}$, i.e., $s=(f, \bar{w})$, with $f=0, \cdots, N_{f}, \bar{w}=1, \cdots, N_{\bar{w}}$ and 
This article has been accepted for inclusion in a future issue of this journal. Content is final as presented, with the exception of pagination.

TABLE IV

MARKet OutComes-SiX-Bus System. POWERS IN MW

\begin{tabular}{|c|c|c|c|c|c|c|}
\hline & \multirow[b]{2}{*}{ Unit } & \multicolumn{4}{|c|}{ Period $t$} \\
\hline & & & 1 & 2 & 3 & 4 \\
\hline \multirow{8}{*}{ Area 1} & \multirow{3}{*}{$P_{i t}$} & G1 & 40.0 & 41.3 & 53.9 & 40.9 \\
\hline & & G2 & 8.7 & 9.2 & 13.4 & 9.1 \\
\hline & & G3 & 85.6 & 89.0 & 95.0 & 87.0 \\
\hline & $P_{w t}$ & WP1 & 1.2 & 6.5 & 11.3 & 3.3 \\
\hline & \multirow{2}{*}{$R_{i t}^{\mathrm{U}}$} & G1 & 0 & 1 & 7.8 & 0.5 \\
\hline & & G3 & 5.7 & 9.5 & 5.0 & 7.5 \\
\hline & \multirow{2}{*}{$R_{i t}^{\mathrm{D}}$} & G1 & 0 & 1.0 & 6.9 & 0.5 \\
\hline & & G3 & 4.3 & 8.5 & 5.3 & 6.5 \\
\hline \multirow{8}{*}{ Area 2} & \multirow{3}{*}{$P_{i t}$} & G4 & 18.7 & 30.0 & 48.0 & 25.9 \\
\hline & & G5 & 0 & 0 & 1.5 & 0 \\
\hline & & G6 & 23.7 & 22.8 & 33.0 & 22.8 \\
\hline & $P_{w t}$ & WP2 & 2.1 & 21.1 & 24.1 & 11.0 \\
\hline & \multirow{2}{*}{$R_{i t}^{\mathrm{U}}$} & G4 & 21.3 & 20.0 & 25.6 & 19.1 \\
\hline & & G6 & 6.2 & 13.1 & 16.8 & 10.1 \\
\hline & \multirow{2}{*}{$R_{i t}^{\mathrm{D}}$} & G4 & 12.5 & 22.8 & 32.0 & 18.7 \\
\hline & & G5 & 0 & 0 & 0.5 & 0 \\
\hline
\end{tabular}

$s=1, \cdots, N_{f} \times N_{\bar{w}}$, where $N_{f}$ is the number of failure incidents including the contingency-free state of the system and $N_{\bar{w}}$ is the number of wind power scenarios. On the assumption that the wind power production and the occurrence of equipment failures are statistically independent, the probability $\pi_{s}$ of each scenario $s$ is computed as $\pi_{s}=\pi_{f} \times \pi_{\bar{w}}$.

The market is centrally cleared based on the above information, and the market outcomes related to dispatched energy and reserve are presented in Table IV.

Note that no load shedding and no wind spillage occur during the entire scheduling horizon under any scenario, and the unexpected disturbances in a given area are covered only by deploying up/down reserve and assistance from the other areas.

Observe that the wind productions are not scheduled to the values that they offer to the market. This scheduling plan, which may be surprising a priori, poses the following relevant question: since the wind production is free, would it not be comparatively more sensible to program wind power productions equal to the offered values? We note that the market schedule for wind generation $\left(P_{w t}\right)$ is different from the actual wind production $\left(P_{w s t}-P_{w s t}^{\text {spill }}\right)$. Roughly speaking, the wind generation schedule is positively correlated with the reserve scheduling so that the market operator can preposition its conventional units in such a way that the corrective action cost is minimum and consequently the wind production in actual operation is maximum. As an example, observe that if the wind productions, scheduled in period 4 , are fixed to their forecast productions, i.e., $30 \mathrm{MW}$, the expected cost rises around $0.1 \%$, which is the result of an $32 \%$ increase in the amount of reserve scheduled in the market.

Next, the decentralized model described in Section II-B is considered. The results of the decentralized model fully coincide with the results of the centralized one after 15 iterations; all quantities of interest (i.e., unit energy and reserve dispatch, tie-line and internal line power flows in both market state and scenario states, etc.) converge within a $\epsilon=10^{-3}$ tolerance to the centralized problem solution values. Fig. 3 illustrates the evolution of the marginal price of border buses 3 and 6, i.e., $\gamma_{33}^{1}$ and $\gamma_{63}^{2}$, at the peak time period (period 3 ) with the number of iterations. It is observed that after some oscillations these variables converge

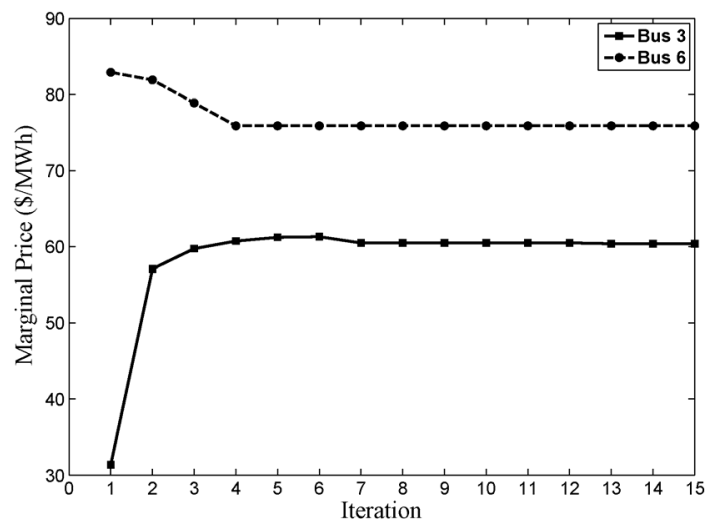

Fig. 3. Evolution of marginal prices of border buses $\gamma_{33}^{1}$ and $\gamma_{63}^{2}$.

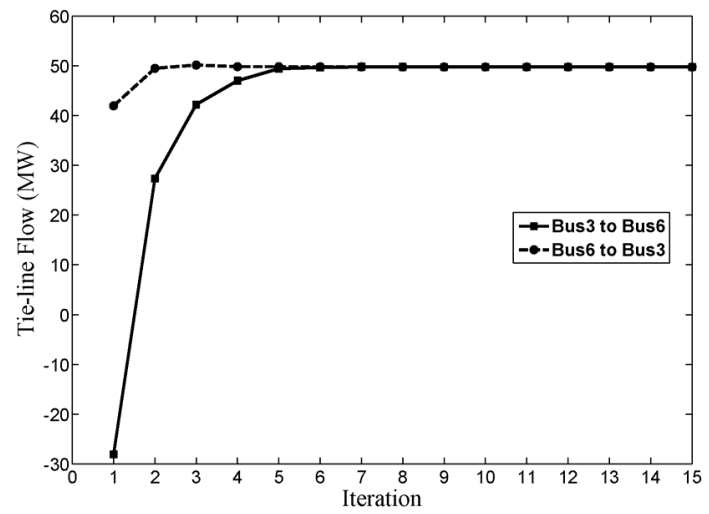

Fig. 4. Evolution of tie-line flow at peak time period.

TABLE V

Comparison of CENTRALIZED AND DeCENTRALIZED Models: SiX-Bus TeST SySTEM

\begin{tabular}{ccc}
\hline & Total Cost $(\$)$ & CPU Time (second) \\
\hline Centralized Model & 38803 & 0.5 \\
\hline Decentralized Model & 38803 & 1.5 \\
\hline
\end{tabular}

to their optimal values. Moreover, note that these prices are different due to the congestion on the tie-line at the peak time period; difference that is called congestion price. It can be inferred from Fig. 3 that marginal prices are higher in areas that mostly import power and lower in areas that export power. In addition, Fig. 4 shows the evolution of the tie-line flow at the peak time period that approach the tie-line capacity limit (i.e., $50 \mathrm{MW}$ ) from both directions during the iterative procedure.

The comparison of total cost and CPU time between the centralized and decentralized models is reported in Table V.

It is relevant to show the benefit of cross border trading in a multi-area interconnected power system. To this end, Figs. 5 and 6 depict the variation of the system total cost and the total share of reserve, respectively, for increasing values of the capacity of the tie-line (0 to $100 \mathrm{MW}$ ). By increasing the tie-line capacity, the congestion is relieved and the cross border trading (energy and reserve sharing) rises to more desirable values. It can be observed in Fig. 6 that the total share of the reserve provided by area 1 (inexpensive area) increases and the share of area 2 (costly area) decreases monotonically as the tie-line capacity increases. As a result, the system total cost reduces as long as the tie-line is not congested (see Fig. 5). 


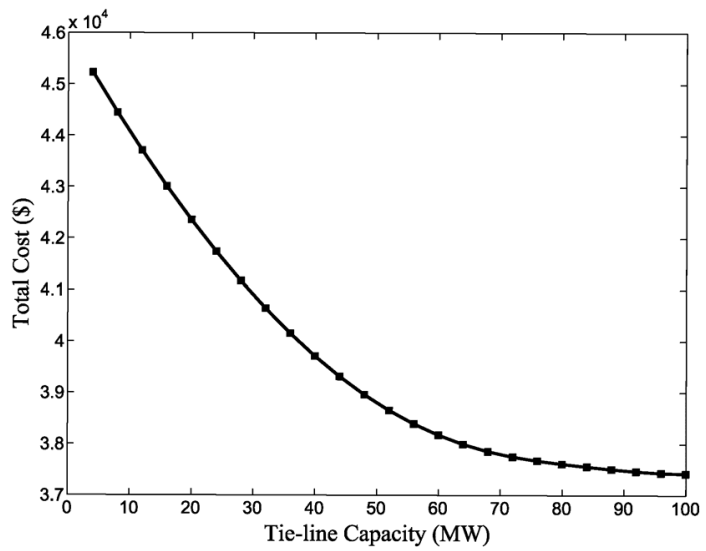

Fig. 5. System total cost versus tie-line capacity.

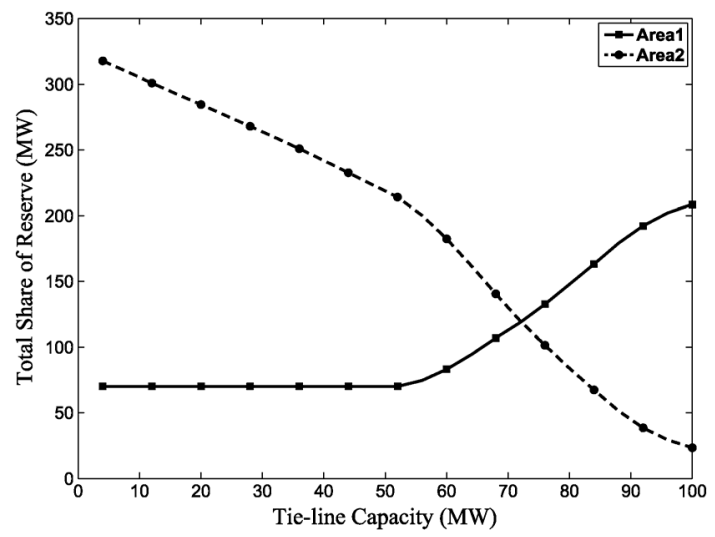

Fig. 6. Total share of reserve versus tie-line capacity.

TABLE VI

FUEL COST DATA

\begin{tabular}{ccccc}
\hline Fuel Type & FO6 & FO2 & Coal & Nuclear \\
\hline Cost (\$/MBTU) & 11.50 & 15.00 & 6.00 & 3.00 \\
\hline
\end{tabular}

\section{B. Three-Area IEEE-RTS}

The proposed decomposition procedure is further tested over a 24-hour scheduling horizon on the 72-bus 3-area IEEE-RTS [32]. Unit cost data are derived from the heat rate data provided in [32] (fitted by quadratic functions) and the fuel cost data listed in Table VI. Each generator offers the maximum possible up/down reserve at a price equal to $10 \%$ of the coefficient $b$ of its cost function. The hourly demand data correspond to the Thursday of winter week 45 with a peak load of $2850 \mathrm{MW}$ for each area ( $8550 \mathrm{MW}$ for the 3-area system). The value of lost load of all demands is assumed to be $1000 \$$ MWh. Two wind farms with $300 \mathrm{MW}$ and $100 \mathrm{MW}$ capacity are located at bus 107 in area 1 and bus 207 in area 2, respectively.

For modeling the most plausible realizations of wind power throughout the scheduling horizon, and in order to make this case study sufficiently realistic, an original set of 276 equally probable wind generation scenarios are considered using publicly available wind power data for year 2012 from two locations in West and East Denmark [33]. Subsequently, to achieve tractability, these scenarios are reduced to 20 by applying the

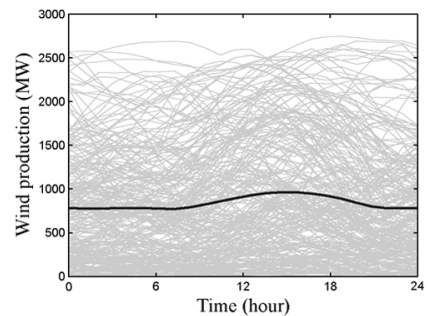

(a)

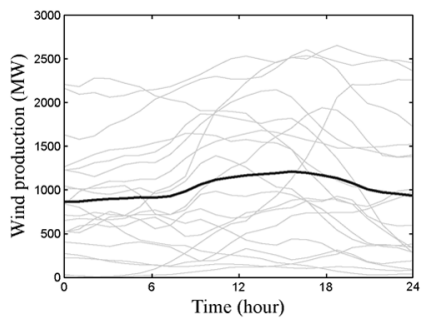

(c)

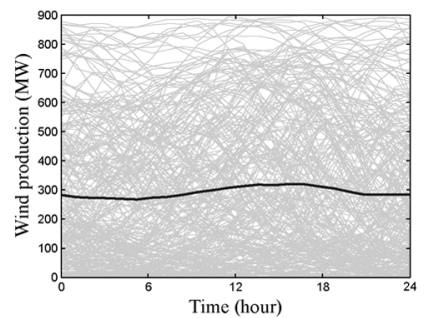

(b)

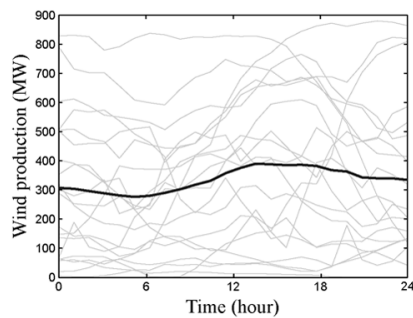

(d)
Fig. 7. The original and the reduced scenarios. (a) Original scenarios of West Denmark wind production. (b) Original scenarios of East Denmark wind production. (c) Reduced scenarios of West Denmark wind production. (d) Reduced scenarios of East Denmark wind production.

TABLE VII

COMPARISON OF CENTRALIZED AND DECENTRALIZED ModELS: THREE-AREA IEEE-RTS

\begin{tabular}{ccc}
\hline & Total Cost $(\$)$ & CPU Time (second) \\
\hline Centralized Model & 7310168 & 287 \\
\hline Decentralized Model $\left(\epsilon=10^{-2}\right)$ & 7326523 & 716 \\
\hline Decentralized Model $\left(\epsilon=10^{-4}\right)$ & 7312049 & 2410 \\
\hline
\end{tabular}

scenario reduction technique proposed in [34]. The original scenario set in Fig. 7(a) and (b) and the corresponding reduced set in Fig. 7(c) and (d) are shown together with their mean values (bold lines). Note that the corresponding wind generation scenarios are scaled down according to the wind farm capacities considered in this case study.

Additionally, we consider a set of credible contingencies: the outage of the nuclear power plant of $400 \mathrm{MW}$ (i.e., the largest unit in the system) located at bus 118 , the outage of the line between bus 103 and bus 124, and the outage of the line between bus 110 and bus 111 , all in area 1 . The remaining elements in the system are assumed to be $100 \%$ reliable and consequently, they never fail. For simplicity and without loss of generality, in this case study we assume that the equipments subject to a possible failure have all the same MTTF of $200 \mathrm{~h}$. The number of scenarios for equipment failures is four, i.e., three contingency scenarios plus the no-contingency scenario. Thus, the total number of scenarios for the market clearing is $80(20 \times 4)$. It is also assumed that the wind generators submit the mean value of scenarios (bold lines) as forecast production for market scheduling.

Table VII provides the total cost and the CPU time for both the centralized and decentralized models for two different values of tolerance. The total cost difference between the centralized and the decentralized solutions are $0.22 \%$ and $0.03 \%$ for tolerances of $\epsilon=10^{-2}$ and $\epsilon=10^{-4}$, respectively. As expected, the smaller value of the tolerance, on one hand, results in better accuracy (i.e., lower cost difference) and, on 
the other hand, requires more iterations to converge. Observe that the CPU time of the decentralized solution with $\epsilon=10^{-4}$ is much higher than that of the decentralized solution with tolerance $\epsilon=10^{-2}$. Needless to say that the CPU time of the decentralized solution is higher than that of the centralized solution in all cases. It is worth noting that as explained in [2], the proposed decentralized model has the property that the area subproblems do not require to attain an optimal solution at each coordinating iteration. It is enough to perform a single solution iteration for each area subproblem. As a consequence, the computation time can be significantly reduced. However, it should be stressed that the main objective of this work is not to attain computational efficiency but preserving the independence of each area.

\section{CONCLUSION}

A multi-area decentralized algorithm is proposed in this paper to carry out an optimal dispatch of energy and reserve in the presence of wind uncertainty and equipment failures. From the theoretical properties of the proposed model and from detailed numerical simulations, the conclusion below are in order:

1) A decentralized solution of the global dispatch is achieved in a decentralized manner while interchanging a moderate level of information.

2) The proposed technique is accurate, as it achieves in a moderate number of iterations the same solution as a centralized algorithm.

3) Area problems do not need to be solved until optimality in every iteration, which may result in high computational performance.

4) The proposed market-clearing model enables cross-border trading through sharing resources (energy and reserve) across boundaries.

5) The proposed model is relevant to the operation of the interconnected European electricity markets.

\section{REFERENCES}

[1] PJM-MISO joint and common market, White paper, Jul. 2005. [Online]. Available: http://www.miso-pjm.com.

[2] A. J. Conejo, E. Castillo, R. Minguez, and R. Garcia-Bertrand, Decomposition Techniques in Mathematical Programming. New York, NY, USA: Springer, 2006

[3] J. Birge and F. Louveaux, Introduction to Stochastic Programming. New York, NY, USA: Springer-Verlag, 1997.

[4] D. Bertsimas and M. Sim, "The price of robustness," Oper. Res., vol. 52, no. 1, pp. 35-53, Jan. 2004.

[5] F. Bouffard, F. D. Galiana, and A. J. Conejo, "Market-clearing with stochastic security—Part I: Formulation," IEEE Trans. Power Syst., vol. 20, no. 4, pp. 1818-1826, Nov. 2005.

[6] F. Bouffard and F. D. Galiana, "Stochastic security for operations planning with significant wind power generation," IEEE Trans. Power Syst., vol. 23, no. 2, pp. 306-316, May 2008.

[7] J. M. Morales, A. J. Conejo, and J. Perez-Ruiz, "Economic valuation of reserves in power systems with high penetration of wind power," IEEE Trans. Power Syst., vol. 24, no. 2, pp. 900-910, May 2009.

[8] P. A. Ruiz, C. R. Philbrick, E. Zak, K. W. Cheung, and P. W. Sauer, "Uncertainty management in the unit commitment problem," IEEE Trans. Power Syst., vol. 24, no. 2, pp. 642-651, May 2009.

[9] A. Papavasiliou, S. S. Oren, and R. P. O'Neill, "Reserve requirements for wind power integration: A scenario-based stochastic programming framework," IEEE Trans. Power Syst., vol. 26, no. 4, pp. 2197-2206, Nov. 2011.
[10] A. Ahmadi Khatir and R. Cherkaoui, "Preventive and corrective security market model," in Proc. 17th Power Systems Computation Conf. (PSCC), Stockholm, Sweden, 2011.

[11] U. A. Ozturk, M. Mazumdar, and B. A. Norman, "A solution to the stochastic unit commitment problem using chance constrained programming," IEEE Trans. Power Syst., vol. 19, no. 3, pp. 1589-1598, Aug. 2004.

[12] Q. Wang, Y. Guan, and J. Wang, “A chance-constrained two-stage stochastic program for unit commitment with uncertain wind power output," IEEE Trans. Power Syst., vol. 27, no. 1, pp. 206-215, Feb. 2012.

[13] D. Pozo and J. Contreras, "A chance-constrained unit commitment with an n-k security criterion and significant wind generation," IEEE Trans. Power Syst., to be published.

[14] D. Bertsimas, E. Litvinov, X. A. Sun, J. Zhao, and T. Zheng, "Adaptive robust optimization for the security constrained unit commitment problem," IEEE Trans. Power Syst., vol. 28, no. 1, pp. 52-63, Feb. 2013.

[15] L. Baringo and A. J. Conejo, "Offering strategy via robust optimization," IEEE Trans. Power Syst., vol. 26, no. 3, pp. 1418-1425, Aug. 2011.

[16] R. Jiang, J. Wang, and Y. Guan, "Robust unit commitment with wind power and pumped storage hydro," IEEE Trans. Power Syst., vol. 27, no. 2, pp. 800-810, May 2012.

[17] A. J. Conejo and J. A. Aguado, "Multi-area coordinated decentralized DC optimal power flow," IEEE Trans. Power Syst., vol. 13, no. 4, pp. 1272-1278, Nov. 1998.

[18] B. H. Kim and R. Baldick, "Coarse-grained distributed optimal power flow," IEEE Trans. Power Syst., vol. 12, no. 2, pp. 932-939, May 1997.

[19] R. Baldick, B. H. Kim, C. Chase, and Y. Luo, "A fast distributed implementation of optimal power flow," IEEE Trans. Power Syst., vol. 14, no. 3, pp. 858-864, Aug. 1999.

[20] F. J. Nogales, F. J. Prieto, and A. J. Conejo, "A decomposition methodology applied to the multi-area optimal power flow problem," Ann. Oper. Res., vol. 120, pp. 99-116, 2003.

[21] N. J. Redondo and A. J. Conejo, "Short-term hydro-thermal coordination by Lagrangian relaxation: Solution of the dual problem," IEEE Trans. Power Syst., vol. 14, no. 1, pp. 89-95, Feb. 1999.

[22] C. Yingvivatanapong, L. Wei-Jen, and E. Liu, "Multi-area power generation dispatch in competitive markets," IEEE Trans. Power Syst., vol. 23, no. 1, pp. 196-203, Feb. 2008.

[23] Q. Jiang, B. Zhou, and M. Zhang, "Parallel augment Lagrangian relaxation method for transient stability constrained unit commitment," IEEE Trans. Power Syst., to be published.

[24] EPEX SPOT: Exchange for the Power Spot Markets, 2012. [Online]. Available: http:/www.epexspot.com.

[25] Nord Pool Spot Market, Annual Report, 2011. [Online]. Available: http://www.nordpoolspot.com.

[26] Market Operator of the Electricity Market of Mainland Spain, OMEL, 2012. [Online]. Available: http://www.omel.es/.

[27] N. Alguacil, A. L. Motto, and A. J. Conejo, "Transmission expansion planning: A mixed-integer LP approach," IEEE Trans. Power Syst., vol. 18, no. 3, pp. 1070-1077, Aug. 2003.

[28] A. J. Conejo, F. J. Nogales, and F. J. Prieto, "A decomposition procedure based on approximate Newton directions," Math. Program., vol. 93, pp. 495-515, 2002.

[29] E. Caro, A. J. Conejo, and R. Minguez, "Decentralized state estimation and bad measurement identification: An efficient Lagrangian relaxation approach," IEEE Trans. Power Syst., vol. 26, no. 4, pp. 2500-2508, Nov. 2011.

[30] R. E. Rosenthal, GAMS-A User's Guide, tech. rep., GAMS Development Corp.. Washington, DC, USA, 2010.

[31] R. Billinton and R. N. Allan, Reliability Evaluation of Power Systems, 2nd ed. New York, NY, USA: Plenum, 1996.

[32] Reliability Test System Task Force, "The IEEE reliability test system-1996," IEEE Trans. Power Syst., vol. 14, no. 3, pp. 1110-1120, Aug. 1999.

[33] Danish Minister for Climate and Energy. [Online]. Available: http:// energinet.dk.

[34] J. M. Morales, S. Pineda, A. J. Conejo, and M. Carrion, "Scenario reduction for futures market trading in electricity markets," IEEE Trans. Power Syst., vol. 24, no. 2, pp. 878-888, May 2009. 


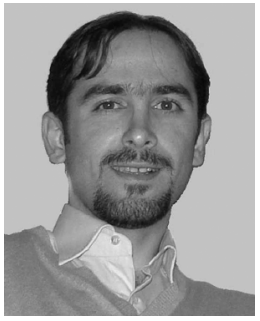

Ali Ahmadi-Khatir (S'09-M'13) received the M.Sc. degree from Sharif University of Technology, Tehran, Iran, in 2005, and the Ph.D. degree from EPFL, Lausanne, Switzerland, in 2013.

His research interests include electricity markets and power system optimization.

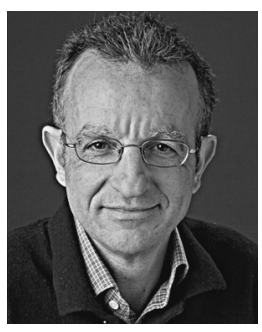

Rachid Cherkaoui (M'05-SM'07) received the M.S. and Ph.D. degrees from EPFL, Lausanne, Switzerland, in 1983 and 1992, respectively.

$\mathrm{He}$ is currently the head of the power system group at EPFL. His research interests include power system operation, planning and electricity market.

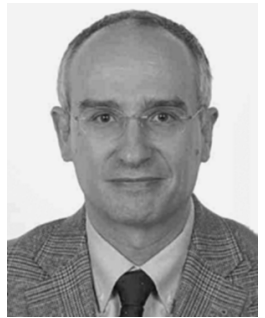

Antonio J. Conejo (F'04) received the M.S. degree from the Massachusetts Institute of Technology, Cambridge, MA, USA, in 1987, and the Ph.D. degree from the Royal Institute of Technology, Stockholm, Sweden, in 1990.

$\mathrm{He}$ is currently a full Professor at the University of Castilla-La Mancha, Ciudad Real, Spain. His research interests include control, operations, planning and economics of electric energy systems, as well as statistics and optimization theory and its applications. 